\title{
Light-induced oligomerization of the transcription factor EL222
}

\author{
Aditya S. Chaudhari ${ }^{1}$, Edel Cunill Semanat ${ }^{1}$, Anne Martel $^{2}$, Bohdan Schneider ${ }^{1}$, Gustavo Fuertes ${ }^{1}$ \\ ${ }^{1}$ Institute of Biotechnology of the Czech Academy of Sciences, Prumyslova 595, 25250 Vestec, Czech Republic, \\ ${ }^{2}$ Institute Laue Langevin, 71 Avenue des Martyrs, 38042 Grenoble CEDEX 9, France
}

Gustavo.fuertes@ibt.cas.cz

\begin{abstract}
Protein self-association is an extremely common phenomenon in biology. However, light-driven protein homo-oligomerization has so far only been described in a few classes of photoreceptors, most notably plant cryptochromes and phytochromes. The characterization of light-induced protein oligomerization is challenging due to the need of synchronizing sample irradiation with data acquisition. Using a combination of carefully chosen methods, we hereby show that EL222, a bacterial transcription factor belonging to the lightoxygen-voltage (LOV) family, can form clusters in a concentration and power dependent manner. In the dark state, the DNA-binding helix-turn-helix module of EL222 is caged by the adjacent LOV domain. Blue-light excitation of the embedded flavin mononucleotide (FMN) cofactor triggers a cascade of protein conformational changes leading to uncaging of the HTH domain, EL222 dimerization, and interaction with its target DNA. Our time-resolved small-angle neutron scattering (SANS) experiments revealed the kinetics of EL222 assembly into high-order oligomers upon illumination and their subsequent disassembly in the dark. The light-induced changes in EL222 size and shape were found to be fully reversible and the photorecovery rate was in line with the well-known FMN photocycle. Further experiments employing fluorescence correlation (FCS) spectroscopy supported the SANS observations and allowed us to gain more insight into the photoinduced oligomerization kinetics. Analyses of the fluorescence traces and FCS curves pointed to the co-existence of multiple diffusing species in EL222 samples illuminated continuously. Moreover, we identified putative protein-protein interaction interfaces and the role of DNA in the aggregation process. Taken together, our hybrid SANS/FCS approach suggests a plausible mechanism of multimer formation in irradiated EL222.
\end{abstract}

\section{Keywords: photosensory receptors; protein aggregation; integrative time-resolved structural biology; small-angle neutron} scattering (SANS); fluorescence correlation spectroscopy (FCS).

This work has been supported by the project "Structural Dynamics of Biomolecular Systems (ELIBIO) (CZ.02.1.01/0.0/0.0/15_003/0000447) from the European Regional Development Fund and the Ministry of Education, Youth and Sports. 